University of Texas Rio Grande Valley

ScholarWorks @ UTRGV

\title{
Effective characteristic polynomials and two-point Pade approximants as summation techniques for the strongly divergent perturbation expansions of the ground state energies of anharmonic oscillators
}

Jiri Cizek

Ernst Joachim Weniger

Paul Bracken

The University of Texas Rio Grande Valley, paul.bracken@utrgv.edu

Vladimir Spirko

Follow this and additional works at: https://scholarworks.utrgv.edu/mss_fac

Part of the Mathematics Commons

\begin{abstract}
Recommended Citation
Čížek, Jiří, Ernst Joachim Weniger, Paul Bracken, and Vladimír Špirko. 1996. “Effective Characteristic Polynomials and Two-Point Padé Approximants as Summation Techniques for the Strongly Divergent Perturbation Expansions of the Ground State Energies of Anharmonic Oscillators." Physical Review E 53 (3): 2925-39. https://doi.org/10.1103/PhysRevE.53.2925.
\end{abstract}

This Article is brought to you for free and open access by the College of Sciences at ScholarWorks @ UTRGV. It has been accepted for inclusion in School of Mathematical and Statistical Sciences Faculty Publications and Presentations by an authorized administrator of ScholarWorks @ UTRGV. For more information, please contact justin.white@utrgv.edu,william.flores01@utrgv.edu. 


\title{
Effective characteristic polynomials and two-point Padé approximants as summation techniques for the strongly divergent perturbation expansions of the ground state energies of anharmonic oscillators
}

\author{
Jiří Č́ížek* \\ Lehrstuhl für Theoretische Chemie, Universität Erlangen-Nürnberg, D-91058 Erlangen, \\ Federal Republic of Germany \\ Ernst Joachim Weniger \\ Institut für Physikalische und Theoretische Chemie, \\ Universität Regensburg, D-93040 Regensburg, Federal Republic of Germany \\ Paul Bracken ${ }^{\dagger}$ \\ Quantum Theory Group, Department of Applied Mathematics, Faculty of Mathematics, \\ University of Waterloo, Waterloo, Ontario, Canada N2L 3G1 \\ Vladimír Špirko \\ J. Heyrovsky Institute of Physical Chemistry, Academy of Sciences of the Czech Republic, \\ Dolejškova 3, 18223 Prague 8, Czech Republic \\ (Received 7 August 1995)
}

\begin{abstract}
Padé approximants are able to sum effectively the Rayleigh-Schrödinger perturbation series for the ground state energy of the quartic anharmonic oscillator, as well as the corresponding renormalized perturbation expansion [E.J. Weniger, J. Č́ižek, and F. Vinette, J. Math. Phys. 34, 571 (1993)]. In the sextic case, Padé approximants are still able to sum these perturbation series, but convergence is so slow that they are computationally useless. In the octic case, Padé approximants are not powerful enough and fail. On the other hand, the inclusion of only a few additional data from the strong coupling domain [E.J. Weniger, Ann. Phys. (N.Y.) (to be published)] greatly enhances the power of summation methods. The summation techniques that we consider are two-point Padé approximants and effective characteristic polynomials. It is shown that these summation methods give good results for the quartic and sextic anharmonic oscillators, and even in the case of the octic anharmonic oscillator, which represents an extremely challenging summation problem, two-point Padé approximants give relatively good results.
\end{abstract}

PACS number(s): 02.70.-c, 03.65-w, 02.30.Lt

\section{THE SUMMATION OF DIVERGENT PERTURBATION EXPANSIONS}

Perturbation theory is one of the few principal methods of approximating solutions to eigenvalue problems in theoretical physics [1-4]. Accordingly, there is an extensive literature. Mathematical aspects of perturbation theory are treated in monographs by Friedrichs [5], Kato [6], Maslov [7], Rellich [8], and in Sec. XII of Reed and Simon [9]. Numerous applications of perturbation theory

\footnotetext{
*Permanent address: Quantum Theory Group, Department of Applied Mathematics, Faculty of Mathematics, University of Waterloo, Waterloo, Ontario, Canada N2L 3G1 and Guelph-Waterloo Centre for Graduate Work in Chemistry, University of Guelph and University of Waterloo, Canada. Also at Quantum Theory Project, Department of Chemistry and Physics, University of Florida, Gainesville, Florida 32611 and at Faculty of Mathematics and Physics, Charles University, Ke Karlovu 3, 12116 Prague 2, Czech Republic.

${ }^{\dagger}$ Present address: Quantum Theory Project, Department of Chemistry and Physics, University of Florida, Gainesville, Florida 32611.
}

are described in books by Adams [10], Arteca, Fernández, and Castro [11], Le Guillou and Zinn-Justin [12], Kumar [13], and Nicolaides, Clark, and Nayfeh [14].

Rayleigh-Schrödinger perturbation theory is designed for systems whose Hamiltonian $\hat{H}(\beta)$ can be partitioned into a completely solvable part $\hat{H}_{0}$ with known eigenvalues and eigenfunctions, and a perturbation $\beta \hat{V}$ according to

$$
\hat{H}(\beta)=\hat{H}_{0}+\beta \hat{V} \text {. }
$$

The formalism of Rayleigh-Schrödinger perturbation theory expresses an eigenvalue $E(\beta)$ of $\hat{H}(\beta)$ as a formal power series in the coupling constant $\beta$ :

$$
E(\beta)=\sum_{m=0}^{\infty} c_{m} \beta^{m}
$$

In the early days of quantum mechanics, the limited computational resources made it normally impossible to compute more than a few terms of a perturbation expansion. Consequently, physically meaningful results could only be obtained for small values of the coupling constant $\beta$, and it did not matter too much whether a perturbation series converges in some neighborhood of $\beta=0$ or 
whether it is only asymptotic as $\beta \rightarrow 0$. Accordingly, convergence questions were largely ignored in the older literature. Because of the dramatic increase of computing power, perturbation expansions can now often be computed to extremely high orders, in particular if symbolic manipulation systems such as MAPLE $[15,16]$ are used. Thus it is now in principle possible to obtain high precision results with the help of perturbation theory and the question whether a perturbation series converges or diverges and what can be done to improve its numerical properties is of greatest practical relevance.

The convergence of the perturbation series (1.2) in a neighborhood of $\beta=0$ is only guaranteed if the domain of $H_{0}$ is contained in the domain of $\hat{V}$,

$$
\mathcal{D}\left(\hat{H}_{0}\right) \subset \mathcal{D}(\hat{V}),
$$

and if the inequality

$$
\|\hat{V} \psi\| \leq a\left\|\hat{H}_{0} \psi\right\|+b\|\psi\|
$$

is satisfied for all $\psi \in \mathcal{D}\left(\hat{H}_{0}\right)$, with $a$ and $b$ being suitable positive constants (compare the Lemma on p. 16, Theorem XII.9 on p. 17, and Theorem XII.11 on p. 21 of Ref. [9], and further references quoted therein). If the perturbation operator $\hat{V}$ does not satisfy conditions (1.3) and (1.4), the perturbation series (1.2) is only asymptotic as $\beta \rightarrow 0$ and diverges. In such a case, summation techniques are needed to give a divergent perturbation series (1.2) any meaning beyond a mere formal expansion.

The summation of divergent series has been a controversial topic in mathematics. The classic books on divergent series are those by Borel [17] and by Hardy [18]. However, there is still a lot of mathematical research going on, as documented by the recently published monographs by Candelpergher, Nosmas, and Pham [19], Ramis [20], Balser [21], and Shawyer and Watson [22].

In many perturbation problems of quantum mechanics or quantum field theory, the perturbation operator $\hat{V}$ does not satisfy conditions (1.3) and (1.4) and the resulting perturbation expansion diverges. Consequently, there is extensive literature on the summation of divergent perturbation expansions. Padé approximants [23-27] have become a standard tool to overcome problems with slowly convergent or divergent power series. Borel's summation method [17] is also of considerable importance. An extensive bibliography on contemporary applications of the Borel method can be found in the book by Shawyer and Watson [22]. Recently, nonlinear sequence transformations have also been used with considerable success for the summation of strongly divergent series [28-37].

The formalism of Rayleigh-Schrödinger perturbation theory produces so-called weak coupling expansions, which are expansions around $\beta=0$. If such an expansion diverges and has to be summed, there is the very annoying problem that summation methods only work well if the coupling constant is small or at most moderately large. If the argument of a divergent power series becomes very large, a straightforward application of a summation technique is not possible because then the terms of the perturbation series diverge individually [38].
These problems in the strong coupling regime can often be overcome with the help of renormalization techniques, which are essentially variable substitutions. In this way, a weak coupling expansion is transformed into a new expansion with hopefully better numerical properties. A discussion of the advantages and disadvantages of various renormalization schemes can be found on pp. 126-135 of [11].

In this article, we want to proceed differently. There are cases in which both weak coupling and strong coupling expansions can be constructed [35,37,39-47]. In such a case, it should be possible to apply summation techniques that use simultaneously information from the weak coupling as well as from the strong coupling expansion. Obviously, such a dual approach should at least in principle be capable of producing better results than a summation technique, which only uses information from either the weak coupling or the strong coupling expansion.

If both a weak coupling and a strong coupling expansion is available, it is an obvious idea to use twopoint Padé approximants as done, for instance, in Refs. [39-41,48,49]. However, other approaches are also possible. In this article, we shall also study effective characteristic polynomials, which were used with considerable success for the description of electron correlation in certain model systems $[42-47,50]$. Since we feel that effective characteristic polynomials should be useful computational tools also in different contexts, we shall use them for the summation of strongly divergent perturbation expansions and compare them with two-point Padé approximants.

Effective characteristic polynomials are not necessarily as efficient as two-point Padé approximants. However, effective characteristic polynomials are in certain cases more flexible than two-point Padé approximants because the symmetry of the exact Hamiltonian can be taken into account in an easy way [43], which may not be possible in the case of two-point Padé approximants [44,39].

In this article, anharmonic oscillators are considered which are defined by the Hamiltonians

$$
\hat{H}^{(m)}(\beta)=\hat{p}^{2}+\hat{x}^{2}+\beta \hat{x}^{2 m}, \quad m=2,3,4, \ldots .
$$

A rigorous analysis of the mathematical properties of these Hamiltonians was done by Simon [51]. Ever since the seminal work of Bender and Wu [52-54], the perturbation expansions of the anharmonic oscillators have been the textbook examples of extremely strongly divergent perturbation expansions.

Harmonic oscillators and their anharmonic counterparts are extremely important model systems in all branches of quantum physics [55] and in particular in quantum field theory. Accordingly, their large order Rayleigh-Schrödinger perturbation expansions are treated in many textbooks on quantum field theory and related topics (see, for instance, pp. 467-473 of Itzykson and Zuber [56], pp. 189-196 and 577-583 of Kleinert [57], pp. 376-382 of Negele and Orland [58], pp. 311313 of Parisi [59], pp. 92-107 of Schulman [60], and pp. 835-847 of Zinn-Justin [61]).

Finally, we would like to emphasize that the goal of this 
article is not the construction of summation techniques, which give extremely accurate results for anharmonic oscillators. Instead, it is our aim to analyze and apply some unconventional and at the same time remarkably simple summation techniques. We want to investigate whether these techniques are, in spite of their simplicity, capable of producing reasonably accurate summation results in the case of the anharmonic oscillators. If this is true, it should be possible to apply these techniques also to systems that are not as well understood as the anharmonic oscillators and are too complicated to permit the use of more sophisticated and therefore computationally more demanding summation techniques.

\section{PERTURBATION EXPANSIONS FOR THE ANHARMONIC OSCILLATORS}

The anharmonicity $x^{2 m}$ with $m=2,3,4, \ldots$ grows for $x \rightarrow \pm \infty$ much faster than the harmonic oscillator potential $x^{2}$. Consequently, there are wave functions $\psi(x)$ belonging to $\mathcal{D}\left(\hat{H}_{0}\right)=\mathcal{D}\left(\hat{p}^{2}\right) \cap \mathcal{D}\left(\hat{x}^{2}\right)$ that do not belong to $\mathcal{D}\left(\hat{x}^{2 m}\right)$. Accordingly, the inequality (1.4) cannot be satisfied by all $\psi \in \mathcal{D}\left(\hat{H}_{0}\right)$ and it is to be expected that the perturbation series

$$
E^{(m)}(\beta)=\sum_{n=0}^{\infty} b_{n}^{(m)} \beta^{n}
$$

for the ground state energy eigenvalue of the Hamiltonian (1.5) diverges for every $\beta \neq 0$. This divergence was confirmed numerically by Bender and $\mathrm{Wu}$ [52-54], who also found that the terms $b_{n}^{(m)}$ behave in the quartic, sextic, and octic case essentially like $n ! / n^{1 / 2},(2 n) ! / n^{1 / 2}$, and $(3 n) ! / n^{1 / 2}$, respectively, as $n \rightarrow \infty$ [compare, for instance, Eqs. (3.5)-(3.7) of Ref. [34]].

The summation of the perturbation series (2.1) is a very difficult problem, in particular in the sextic and octic case and for large values of $\beta$. There is an extensive literature on the Padé summation of this perturbation series. In the quartic and sextic cases, it could be proven rigorously that the perturbation series (2.1) is a Stieltjes series and that Padé approximants are able to sum it $[51,62]$. In the quartic case, Padé approximants give satisfactory results if the coupling constant is small. The sextic anharmonic oscillator seems to be a borderline case since Padé approximants converge so slowly that they are computationally useless $[51,62,63]$. In the octic case, it was proven rigorously by Graffi and Grecchi [64] that Padé approximants are not able to sum the perturbation series (2.1).

With the help of Symanzik scaling, which is described in Sec. II. 2 of Ref. [51] or in Sec. II of Ref. [65], it can be shown that the ground state energy eigenvalue $E^{(m)}(\beta)$ of the Hamiltonian (1.5) possesses also a strong coupling expansion

$$
E^{(m)}(\beta)=\beta^{1 /(m+1)} \sum_{n=0}^{\infty} K_{n}^{(m)} \beta^{-2 n /(m+1)} .
$$

Simon proved that this series converges in the quartic case if $\beta$ is sufficiently large (Lemma II.1 on p. 81 and Theorem II.1.2 on p. 82 of Ref. [51]). The extension of Simon's convergence proof to $m=3,4, \ldots$ is straightforward (see Theorems 10-1 and 10-2 of Ref. [35]). Hence the use of the perturbation series (2.2) in the troublesome strong coupling regime is in principle highly desirable. However, the strong coupling expansion (2.2) cannot be used for all physically relevant $\beta \in[0, \infty)$ since the powers of $\beta^{-2 /(m+1)}$ become singular as $\beta \rightarrow 0$. Moreover, the infinite series on the right-hand side of Eq. (2.2) has to approach plus infinity as $\beta \rightarrow 0$ in order to satisfy the normalization condition $E^{(m)}(0)=1$.

It follows from the strong coupling expansion (2.2) that the energy eigenvalue $E^{(m)}(\beta)$ behaves in the strong coupling regime like a fractional power of $\beta$ :

$$
E^{(m)}(\beta) \sim \beta^{1 /(m+1)}, \quad \beta \rightarrow \infty .
$$

A summation method has to be able to model this fractional power behavior in order to be effective in the strong coupling regime. In the case of the perturbation series (2.1), there is no easy way of extracting the fractional power behavior (2.3) from a finite number of terms or partial sums. Accordingly, it is extremely difficult to sum this series effectively if $\beta$ is large. In contrast, the terms and partial sums of the strong coupling expansion (2.2) automatically possess the correct asymptotic behavior (2.3) as $\beta \rightarrow \infty$ and a few terms suffice to compute $E^{(m)}(\beta)$ accurately in the strong coupling regime (compare Table II of Ref. [66]).

The main disadvantage of the strong coupling expansion (2.2) is that the computation of the coefficients $K_{n}^{(m)}$ is very difficult. Symanzik scaling replaces the Hamiltonian (1.5) by a strong coupling Hamiltonian

$$
\begin{aligned}
& \beta^{1 /(m+1)} \hat{H}_{\mathrm{sc}}^{(m)}(\beta) \\
& =\beta^{1 /(m+1)}\left[\hat{p}^{2}+\beta^{-2 /(m+1)} \hat{x}^{2}+\hat{x}^{2 m}\right],
\end{aligned}
$$

which can be partitioned into an unperturbed Hamiltonian $\hat{p}^{2}+\hat{x}^{2 m}$ and a perturbation $\beta^{-2 /(m+1)} \hat{x}^{2}$ (more details can be found in Sec. 10.3 of Ref. [35] or in Refs. $[37,51])$. However, the eigenvalues and eigenfunctions of the unperturbed Hamiltonian $\hat{p}^{2}+\hat{x}^{2 m}$ are not known and the usual formalism of Rayleigh-Schrödinger perturbation theory for the computation of the coefficients of the perturbation series cannot be used.

There are alternative approaches for the computation of the coefficients $K_{n}^{(m)}[66-72]$. In the quartic case, the best results were obtained by Janke and Kleinert [66]. Other good results were obtained by Guardiola, Solís, and Ros [71], who were able to compute coefficients of the strong coupling expansion (2.2) for the ground state energy of an anharmonic oscillator with an $\hat{x}^{4}, \hat{x}^{6}, \hat{x}^{8}$, and even $\hat{x}^{10}$ anharmonicity. Nevertheless, the computation of the coefficients $K_{n}^{(m)}$ remains in particular for larger values of $m$ a very difficult problem, and further progress would be highly desirable.

The perturbation series $(2.1)$ is a power series in $\beta$ and the strong coupling expansion (2.2) is a power series in $\beta^{-2 /(m+1)}$. Consequently, these two expansions have incompatible variables and cannot be used to provide si- 
multaneously information from the weak coupling and strong coupling regime to effective characteristic polynomials and two-point Padé approximants, which will be discussed in the following sections.

Alternative perturbation expansions, which are suited for our purposes, can be constructed with the help of a renormalization scheme which was first used by Čížek and Vrscay [73] in the case of the quartic and sextic anharmonic oscillator, and which was worked out in full detail in an article by Vinette and Č́žek [74]. This renormalization scheme was first used for the construction of rational approximants yielding upper and lower bounds for the ground state energy $E^{(m)}(\beta)$ of the quartic, sextic, and octic anharmonic oscillator [74]. Later, it was used to facilitate the summation of perturbation expansions of anharmonic oscillators with the help of sequence transformations [31,33-37] and also for the computation of energy eigenvalues of anharmonic oscillators with the help of an iterative scheme based on the generalized Bloch equation [75].

The renormalization scheme of Vinette and Cížek [74] may be viewed as a variable substitution that replaces the coupling constant $\beta$ by a renormalized coupling constant $\kappa$ according to [Eq. (3.19) of Ref. [34]]

$$
\beta=\frac{1}{B_{m}} \frac{\kappa}{(1-\kappa)^{(m+1) / 2}}, \quad m=2,3,4,
$$

where

$$
B_{m}=\frac{m(2 m-1) ! !}{2^{m-1}}, \quad m=2,3,4 .
$$

It follows from Eq. (2.5) that the semi-infinite interval $[0, \infty)$ for $\beta$ is mapped onto the unit interval $[0,1)$ for $\kappa$. Moreover, $\beta=0$ corresponds to $\kappa=0$ and $\beta \rightarrow \infty$ to $\kappa \rightarrow 1$.

The renormalization scheme of Vinette and Č́žek [74], which is also based on Symanzik scaling, replaces the Hamiltonian (1.5) by a renormalized Hamiltonian (compare, for instance, Sec. 4 of Ref. [37])

$$
\begin{aligned}
& (1-\kappa)^{-1 / 2} \hat{\mathcal{H}}^{(m)}(\kappa) \\
& =(1-\kappa)^{-1 / 2}\left(\hat{p}^{2}+\hat{x}^{2}+\frac{\kappa}{B_{m}}\left[\hat{x}^{2 m}-B_{m} \hat{x}^{2}\right]\right) .
\end{aligned}
$$

Scaling is a unitary transformation [51,65]. Consequently, the eigenvalues of the Hamiltonians (1.5) and $(2.7)$ are identical and the ground state energy $E^{(m)}(\beta)$ is partitioned into a prefactor $(1-\kappa)^{-1 / 2}$ multiplied by a renormalized ground state energy $E_{R}^{(m)}(\kappa)$ [Eq. (3.30) of Ref. [34]]:

$$
E^{(m)}(\beta)=(1-\kappa)^{-1 / 2} E_{R}^{(m)}(\kappa) .
$$

It also follows from Eq. (2.5) that

$$
\beta^{1 /(m+1)} \sim(1-\kappa)^{-1 / 2}
$$

as $\beta \rightarrow \infty$. Thus $E_{R}^{(m)}(\kappa)$ remains finite as $\kappa \rightarrow 1$, whereas $(1-\kappa)^{-1 / 2}$ diverges as $\kappa \rightarrow 1$ like $\beta^{1 /(m+1)}$ as $\beta \rightarrow \infty$.
The renormalized Hamiltonian $\hat{\mathcal{H}}^{(m)}(\kappa)$ can be partitioned into an unperturbed Hamiltonian $\hat{p}^{2}+\hat{x}^{2}$ and a perturbation $\kappa\left[\left(\hat{x}^{2 m} / B_{m}\right)-\hat{x}^{2}\right]$, which implies that $E_{R}^{(m)}(\kappa)$ possesses the following perturbation series in $\kappa$ [Eq. (3.31) of Ref. [34]]:

$$
E_{R}^{(m)}(\kappa)=\sum_{n=0}^{\infty} c_{n}^{(m)} \kappa^{n}
$$

The computation of the coefficients $c_{n}^{(m)}$ is described in the Appendix of Ref. [34]. In the quartic, sextic, and octic case, the coefficients $c_{n}^{(m)}$ grow similarly to the coefficients $b_{n}^{(m)}$ of the perturbation series (2.1) essentially like $n ! / n^{1 / 2},(2 n) ! / n^{1 / 2}$, and $(3 n) ! / n^{1 / 2}$, respectively, as $n \rightarrow \infty$ [compare Eqs. (3.33)-(3.38) of Ref. [34]].

Thus the renormalized perturbation series (2.10) diverges quite strongly for every $\kappa \neq 0$ and it looks as if the main achievement of the renormalization scheme of Vinette and Č́žek [74] would be the bounded domain of the renormalized coupling constant $\kappa$. If, however, the ground state energy $E^{(m)}(\beta)$ is expressed via Eqs. (2.8) and (2.10) according to [Eq. (5.3) of Ref. [34]]

$$
E^{(m)}(\beta)=(1-\kappa)^{-1 / 2} \sum_{n=0}^{\infty} c_{n}^{(m)} \kappa^{n},
$$

the prefactor $(1-\kappa)^{-1 / 2}$ guarantees that the terms and partial sums of this perturbation series possess automatically the correct asymptotic behavior (2.3) as $\beta \rightarrow \infty$. This has far-reaching numerical consequences. A very convincing example is the infinite coupling limit

$$
k_{m}=\lim _{\beta \rightarrow \infty} E^{(m)}(\beta) / \beta^{1 /(m+1)},
$$

which is identical to the ground state eigenvalue of the Hamiltonian $\hat{p}^{2}+\hat{x}^{2 m}$ and with the leading term $K_{0}^{(m)}$ of the strong coupling expansion (2.2). The infinite coupling limit $k_{m}$ cannot be computed by a straightforward summation of the weak coupling expansion (2.1). However, it can be computed comparatively easily via the perturbation series (2.11), since one only has to sum the divergent series [Eq. (3.44) of Ref. [34]]

$$
k_{m}=\left[B_{m}\right]^{1 /(m+1)} \sum_{n=0}^{\infty} c_{n}^{(m)}
$$

With the help of the sequence transformation $\delta_{k}^{(n)}\left(\zeta, s_{n}\right)$, which is defined in Eq. (8.4-4) of Ref. [28], this series can be summed efficiently and in particular $k_{2}$ and $k_{3}$ can be computed with remarkable accuracy (compare Tables I-III of Ref. [31], Table 2 of Ref. [33], and Tables VII-IX of Ref. [34]).

Moreover, the results in Tables IV-VI of Ref. [34], Tables 10-9 through 10-11 of Ref. [35], Tables 1 and 2 of Ref. [36], and Table 1 of Ref. [37] show that the renormalized perturbation series (2.11) can also for nonzero but finite values of $\beta$ be summed much more effectively than the perturbation series (2.1). Particularly good summation 
results were again obtained with the help of the sequence transformation $\delta_{k}^{(n)}\left(\zeta, s_{n}\right)$.

The summation results mentioned above show that the renormalization scheme of Vinette and Ćížek [74] is extremely useful in the context of perturbation theory. However, it can also be very helpful in variational calculations. In Table I, we determine variationally for different values of $\beta$ approximations to the ground state eigenvalues of the Hamiltonians

$$
\hat{H}^{(2)}(\beta)=\hat{p}^{2}+\hat{x}^{2}+\beta \hat{x}^{4}
$$

and

$$
\begin{aligned}
& (1-\kappa)^{-1 / 2} \hat{\mathcal{H}}^{(2)}(\kappa) \\
& \quad=(1-\kappa)^{-1 / 2}\left\{\hat{p}^{2}+\hat{x}^{2}+\frac{\kappa}{3}\left[\hat{x}^{4}-3 \hat{x}^{2}\right]\right\} .
\end{aligned}
$$

Since these two Hamiltonians are connected by Symanzik scaling, their eigenvalues are identical. However, in particular for large values for $\beta$, the numerical properties of the two Hamiltonians differ substantially.

As basis functions we use in both cases the eigenfunctions of the harmonic oscillator, which are defined by

$$
\psi_{n}(x)=\left[2^{n} n ! \pi^{1 / 2}\right]^{-1 / 2} e^{-x^{2} / 2} H_{n}(x) .
$$

Here $H_{n}(x)$ is a Hermite polynomial.

The ground state eigenfunctions of the Hamiltonians (1.5) and (2.7) are even. Consequently, only eigenfunctions $\psi_{n}(x)$ with even indices $n$ have to be used in the variational calculation. In Table I the trial function

$$
\Psi^{(4)}(x)=\sum_{\nu=0}^{4} c_{\nu} \psi_{2 \nu}(x)
$$

was used, which contains five linear variational parameters. The "exact" results in Table I were obtained from Table I of Ref. [74]. For a given value of $\beta$, the corresponding renormalized coupling constant $\kappa$ was computed by solving Eq. (2.5) numerically with the help of the MAPLE command fsolve (see p. 97 of Ref. [16]).

The results in Table I show that the ground state eigenvalue of the Hamiltonian $\hat{H}^{(2)}(\beta)$ can only be determined accurately with the help of the trial function (2.17) if $\beta$ is small or at most moderately large. If $\beta$ is large, the anharmonicity $\hat{x}^{4}$ dominates and the trial function (2.17) is no longer able to provide a sufficiently accu-

TABLE I. Variational determination of the ground state energy $E^{(2)}(\beta)$ of the quartic anharmonic oscillator via the Hamiltonian $\hat{H}^{(2)}(\beta)$, Eq. (2.14), and the renormalized Hamiltonian $(1-\kappa)^{-1 / 2} \hat{\mathcal{H}}^{(2)}(\kappa)$, Eq. $(2.15)$.

\begin{tabular}{lrrr}
\hline$\beta$ & $\hat{H}^{(2)}(\beta)$ & \multicolumn{1}{c}{$\hat{\mathcal{H}}^{(2)}(\kappa)$} & \multirow{2}{*}{ Exact } \\
\hline 0.2 & & 1.118294 & 1.118293 \\
1.0 & 1.118293 & 1.392375 & 1.392352 \\
4.0 & 1.393371 & 1.903219 & 1.903137 \\
100.0 & 1.909023 & 4.999800 & 4.999418 \\
2000.0 & 8.084644 & 13.389537 & 13.388442 \\
\hline \hline
\end{tabular}

rate approximation to the ground state eigenfunction of the Hamiltonian $\hat{H}^{(2)}(\beta)$. In contrast, the trial function (2.17) gives good variational results for the renormalized Hamiltonian $(1-\kappa)^{-1 / 2} \hat{\mathcal{H}}^{(2)}(\kappa)$ even if $\beta$ is very large. Loosely speaking, we may say that $(1-\kappa)^{-1 / 2} \hat{\mathcal{H}}^{(2)}(\kappa)$ is in the strong coupling regime closer to the unperturbed Hamiltonian $\hat{p}^{2}+\hat{x}^{2}$ than $\hat{H}^{(2)}(\beta)$.

The renormalized Hamiltonian $\hat{\mathcal{H}}^{(m)}(\kappa)$, which is defined in Eq. (2.7), can also be partitioned into an unperturbed Hamiltonian $\hat{p}^{2}+\hat{x}^{2 m} / B_{m}$ and a perturbation $(1-\kappa)\left[\hat{x}^{2}-\hat{x}^{2 m} / B_{m}\right]$ [Eqs. (5.1)-(5.3) of Ref. [37]]. Consequently, $E_{R}^{(m)}(\kappa)$ possesses also a perturbation series in $1-\kappa$ [Eq. (5.4) of Ref. [37]]:

$$
E_{R}^{(m)}(\kappa)=\sum_{n=0}^{\infty} \Gamma_{n}^{(m)}(1-\kappa)^{n} .
$$

Since $\kappa \rightarrow 1$ corresponds to $\beta \rightarrow \infty$, it follows from Eq. (2.8) that $E^{(m)}(\beta)$ possesses a renormalized strong coupling expansion [Eq. (5.5) of Ref. [37]] that becomes trivial as $\beta \rightarrow \infty$ :

$$
E^{(m)}(\beta)=(1-\kappa)^{-1 / 2} \sum_{n=0}^{\infty} \Gamma_{n}^{(m)}(1-\kappa)^{n} .
$$

The coefficients $\Gamma_{n}^{(m)}$ of the expansions (2.18) and (2.19) are defined by the divergent series [Eq. (5.12) of Ref. [37]]

$$
\Gamma_{n}^{(m)}=\frac{(-1)^{n}}{n !} \sum_{\nu=0}^{\infty}(\nu+1)_{n} c_{n+\nu}^{(m)} .
$$

In Tables 2-4 of Ref. [37], the coefficients $\Gamma_{0}^{(2)}, \Gamma_{1}^{(2)}, \ldots$, $\Gamma_{20}^{(2)}, \Gamma_{0}^{(3)}, \Gamma_{1}^{(3)}, \ldots, \Gamma_{9}^{(3)}$, and $\Gamma_{0}^{(4)}, \Gamma_{1}^{(4)}, \ldots, \Gamma_{5}^{(4)}$ are listed, which were computed by summing the divergent series (2.20) with the help of the sequence transformation $\delta_{k}^{(n)}\left(\zeta, s_{n}\right)$ [Eq. (8.4-4) of Ref. [28]].

In Theorems 1 and 2 of Ref. [37] it was proven that the renormalized energy $E_{R}^{(m)}(\kappa)$ is analytic in a neighborhood of $\kappa=1$. This implies that the perturbation series (2.18) for $E_{R}^{(m)}(\kappa)$ converges in a neighborhood of $\kappa=1$ or, equivalently, that the renormalized strong coupling expansion (2.19) for $E^{(m)}(\beta)$ converges for sufficiently large $\beta$. Moreover, there is strong numerical evidence that the perturbation series (2.18) converges for all $\kappa \in[0,1]$, which implies that the renormalized strong coupling expansion (2.19) converges for all physically relevant $\beta \in[0, \infty)$ (compare Tables $5-8$ of Ref. [37]).

Unlike the perturbation expansion (2.1) and the strong coupling expansion (2.2), the two renormalized perturbation expansions (2.11) and (2.19) have compatible variables. Therefore, they are able to provide numerically useful information from the weak coupling and the strong coupling regime simultaneously to effective characteristic polynomials and two-point Padé approximants.

In all calculations involving the renormalized perturbation expansions (2.11) and (2.19), we retain the prefactor $(1-\kappa)^{-1 / 2}$ since it guarantees that our summation results automatically have the correct asymptotic behavior 
(2.3) as $\beta \rightarrow \infty$. Consequently, we essentially try to determine the renormalized energy $E_{R}^{(m)}(\kappa)$, using a certain number of input data from the weak coupling expansion (2.10) around $\kappa=0$ as well as from the strong coupling expansion (2.18) around $\kappa=1$.

\section{EFFECTIVE CHARACTERISTIC POLYNOMIALS AS A SUMMATION TECHNIQUE}

Let us assume that we try to determine an approximation to the ground state eigenvalue of the Hamiltonian (1.1) variationally. If the trial function is a linear combination of $n$ orthonormal basis functions $\Phi_{\nu}$ with $\nu=1,2, \ldots, n$, the secular problem

$$
\operatorname{det}\left|\left\langle\Phi_{\mu}|\hat{H}(\beta)| \Phi_{\nu}\right\rangle-E \delta_{\mu \nu}\right|=0
$$

results, which is a polynomial $P_{n}(E)$ of degree $n$ in the unknown energy $E$. It can be shown easily that the resulting characteristic polynomial in $E$ has in the case of the Hamiltonian (1.1) the general structure

$$
P_{n}(E)=\sum_{k=0}^{n} E^{k} \sum_{j=0}^{n-k} f_{j}^{k} \beta^{j}=0 .
$$

The coefficients $f_{j}^{k}$ can be constructed from the matrix elements $\left\langle\Phi_{\mu}|\hat{H}(\beta)| \Phi_{\nu}\right\rangle=\left\langle\Phi_{\mu}\left|\hat{H}_{0}\right| \Phi_{\nu}\right\rangle+\beta\left\langle\Phi_{\mu}|\hat{V}| \Phi_{\nu}\right\rangle$. Obviously, we must have $f_{0}^{n}=1$ for all $n \geq 1$.

A reasonable accuracy can only be achieved if the dimensionality $n$ of a variational calculation is sufficiently large. Alternatively, one could use small values of $n$ and replace the Hamiltonian (1.1) by a suitably chosen effective Hamiltonian leading to an effective characteristic polynomial $P_{n}(E)$ of the same general structure as the one in Eq. (3.2).

In this article we choose the coefficients $f_{j}^{k}$ of the effective characteristic polynomial in such a way that the Taylor expansion of the effective characteristic polynomial around $\beta=0$ reproduces the Taylor expansion of the characteristic polynomial of the exact Hamiltonian (1.1) up to a given order.

This can be accomplished by substituting the perturbation expansion (1.2) for the energy into the characteristic polynomial (3.2) of the effective Hamiltonian, yielding

$$
\begin{gathered}
P_{n}\left(\sum_{m=0}^{\infty} c_{m} \beta^{m}\right) \\
=\sum_{k=0}^{n}\left(\sum_{m=0}^{\infty} c_{m} \beta^{m}\right)^{k} \sum_{j=0}^{n-k} f_{j}^{k} \beta^{j}=0
\end{gathered}
$$

For a fixed value of $n$, the effective characteristic polynomial (3.2) contains $(n+1)(n+2) / 2$ coefficients $f_{j}^{k}$. Because of $f_{0}^{n}=1$, only $n(n+3) / 2$ of these coefficients are unspecified and have to be determined. For that purpose, we compute symbolically the Taylor expansion of the substituted characteristic polynomial (3.3) around $\beta=0$ including terms up to the order $n(n+3) / 2-1$ in $\beta$ :

$$
\begin{aligned}
& P_{n}\left(\sum_{m=0}^{\infty} c_{m} \beta^{m}\right) \\
& =\sum_{t=0}^{n(n+3) / 2-1} A_{t} \beta^{t}+O\left(\beta^{n(n+3) / 2}\right) .
\end{aligned}
$$

This can be done conveniently with the help of the MAPLE commands taylor and coeff (see pp. 213 and 19, respectively, of Ref. [16]). Then, we set the coefficients $A_{t}$ with $0 \leq t \leq n(n+3) / 2-1$ of this Taylor expansion equal to zero. This leads to a system of $n(n+3) / 2$ linear equations $A_{t}=0$ for the unspecified coefficients $f_{j}^{k}$, which is solved symbolically with the help of the MAPLE command solve (see p. 200 of Ref. [16]).

In this way, we can construct the effective characteristic polynomial (3.2) with unspecified energy eigenvalue $E$ and unspecified coupling constant $\beta$. After substituting the desired numerical value of $\beta$ into the effective characteristic polynomial (3.2), we determine its roots numerically with the help of the MAPLE command fsolve (see p. 97 of Ref. [16]).

Thus our approach relies heavily on the symbolic manipulation language MAPLE $[15,16]$ both for the construction of the system of linear equations leading to the effective characteristic polynomial (3.2) as well as for the numerical determination of its roots. This extensive use of MAPLE is both an advantage as well as a disadvantage. With the help of MAPLE, we are able to perform complicated mathematical operations symbolically, i.e., free of rounding errors. Since, however, the symbolic solution of large systems of nonlinear equations is quite demanding with respect to computer time and even more so with respect to memory, the complexity of our calculations is very much limited by the available computer resources. Consequently, our approach seems to be particularly suited if only relatively few terms of a perturbation series are available.

Another potential problem of our approach is the numerical determination of the roots of the effective characteristic polynomial with the help of the MAPLE command fsolve. As is well known, the numerically determined roots of a polynomial can be extremely susceptible to small perturbations of the polynomial coefficients (compare, for instance, the example on p. 305 of Ref. [76]). Accordingly, we observed that the accuracy of our results depended in some cases quite strongly on the accuracy of our input data.

It is important to note that we reconstruct only the characteristic polynomial of the effective Hamiltonian but not the effective Hamiltonian itself. If we would instead try to reconstruct the effective Hamiltonian, we would end up with a complicated system of nonlinear equations.

The approach described above can be used to sum either the perturbation series (2.1) or the renormalized perturbation series (2.11), which are both weak coupling expansions. The only difference is that in the case of the renormalized series the prefactor $(1-\kappa)^{-1 / 2}$ is retained. 
Thus we consider effective characteristic polynomials in the renormalized ground state energy $E_{R}^{(m)}(\kappa)$,

$$
\mathcal{P}_{n}\left(E_{R}^{(m)}\right)=\sum_{\nu=0}^{n}\left(E_{R}^{(m)}\right)^{\nu} \sum_{j=0}^{n-\nu} \mathcal{F}_{j}^{\nu} \kappa^{j}=0
$$

which have formally the same structure as the characteristic polynomials (3.2). Again, we must have $\mathcal{F}_{0}^{n}=1$ for all $n \geq 1$.

Next, we replace $E_{R}^{(m)}(\kappa)$ by its perturbation series (2.10), yielding

$$
\begin{aligned}
\mathcal{P}_{n}\left(\sum_{\mu=0}^{\infty} c_{\mu}^{(m)} \kappa^{\mu}\right) \\
=\sum_{\nu=0}^{n}\left(\sum_{\mu=0}^{\infty} c_{\mu}^{(m)} \kappa^{\mu}\right)^{\nu} \sum_{j=0}^{n-\nu} \mathcal{F}_{j}^{\nu} \kappa^{j}=0
\end{aligned}
$$

and compute a Taylor expansion of the substituted characteristic polynomial around $\kappa=0$ symbolically, including terms up to the order $n(n+3) / 2-1$ in $\kappa$ :

$$
\begin{aligned}
& \mathcal{P}_{n}\left(\sum_{\mu=0}^{\infty} c_{\mu}^{(m)} \kappa^{\mu}\right) \\
& =\sum_{t=0}^{n(n+3) / 2-1} \mathcal{A}_{t} \kappa^{t}+O\left(\kappa^{n(n+3) / 2}\right) .
\end{aligned}
$$

This leads to a system of $n(n+3) / 2$ equations $\mathcal{A}_{t}=0$ for the unspecified coefficients $\mathcal{F}_{j}^{\nu}$ in Eq. (3.5).

In this way, the effective characteristic polynomial $\mathcal{P}_{n}\left(E_{R}^{(m)}\right)$ is constructed symbolically with unspecified renormalized energy and unspecified $\kappa$. Then, the numerical value of $\kappa$ is computed for a given value of $\beta$ by solving Eq. (2.5) numerically with the help of the MAPLE command fsolve and substituted in the effective characteristic polynomial. In addition, $E_{R}^{(m)}(\kappa)$ is replaced by $(1-\kappa)^{1 / 2} E^{(m)}(\beta)$ according to Eq. (2.8). Finally, the roots of the resulting characteristic polynomial in $E^{(m)}(\beta)$ are determined numerically using fsolve.

In Table II both the perturbation series (2.1) as well as the renormalized perturbation series (2.11) for the ground state energy $E^{(2)}(\beta)$ of the quartic anharmonic oscillator is summed by effective characteristic polynomials of degrees $n=3$ and $n=4$, respectively. The exact results in Table II were obtained from Table I of Ref. [74].

As mentioned above, an effective characteristic polynomial with degree $n$ of the type of Eq. (3.2) or (3.5) contains $n(n+3) / 2$ unspecified coefficients. Hence, for $n=3$ and $n=4$, only the coefficients $b_{\nu}^{(2)}$ and $c_{\nu}^{(2)}$ of the perturbation series (2.1) and (2.11) with $0 \leq \nu \leq 8$ and $0 \leq \nu \leq 13$, respectively, are needed for the construction of the effective characteristic polynomials.

The results in Table II show once more that the summation of the perturbation series (2.1) in the strong coupling regime is very difficult. In contrast, the summation of the renormalized perturbation series (2.11) by effective characteristic polynomials gives very good summation results even if $\beta$ is very large.

In order to be able to estimate the quality of our summation results for the ground state energy $E^{(2)}(\beta)$ of the quartic anharmonic oscillator, we transform the partial sums of the perturbation series (2.1) and (2.11), respectively, into Padé approximants. To make our Padé results comparable with the summation results in Table II, we consider only those Padé approximants that use at most the coefficients $b_{\nu}^{(2)}$ and $c_{\nu}^{(2)}$ with $0 \leq \nu \leq 14$.

We do the Padé summations as we did it in Ref. [34]: The Padé approximants are computed with the help of Wynn's $\epsilon$ algorithm [77]. Moreover, we only sum the perturbation series for the energy shifts $\Delta E^{(2)}(\beta)$ and $(1-\kappa)^{-1 / 2} \Delta E_{R}^{(2)}(\kappa)$, which are defined by $E^{(2)}(\beta)=$ $1+\Delta E^{(2)}(\beta)$ and $E^{(2)}(\beta)=(1-\kappa)^{-1 / 2}\left[1+\Delta E_{R}^{(2)}(\kappa)\right]$, respectively. Consequently, the input data for Wynn's $\epsilon$ algorithm [77] are the partial sums

$$
s_{n}^{(m)}(\beta)=\sum_{j=0}^{n} b_{j+1}^{(m)} \beta^{j},
$$

with $m=2$ of the perturbation series for the energy shift $\Delta E^{(2)}(\beta)$, and the partial sums

$$
\sigma_{n}^{(m)}(\kappa)=(1-\kappa)^{-1 / 2} \sum_{j=0}^{n} c_{j+1}^{(m)} \kappa^{j},
$$

with $m=2$ of the perturbation series for the renormalized energy shift $(1-\kappa)^{-1 / 2} \Delta E_{R}^{(2)}(\kappa)$.

In Table III the Padé approximants $1+\beta[6 / 6]$ and $1+\beta[7 / 6]$ obtained from the series $(2.1)$ and the Padé approximants $(1-\kappa)^{-1 / 2}(1+\kappa[6 / 6])$ and $(1-\kappa)^{-1 / 2}(1+$ $\kappa[7 / 6])$ obtained from the renormalized perturbation series $(2.11)$ are listed, which use only the coefficients $b_{\nu}^{(2)}$ and $c_{\nu}^{(2)}$ with $0 \leq \nu \leq 14$

A comparison of Tables II and III shows that the renormalized perturbation series (2.11) can again be summed

\begin{tabular}{|c|c|c|c|c|c|}
\hline \multirow[b]{2}{*}{$\beta$} & \multicolumn{2}{|c|}{ Perturbation series (2.1) } & \multicolumn{2}{|c|}{ Renormalized perturbation series (2.11) } & \multirow[b]{2}{*}{ Exact } \\
\hline & $n=3$ & $n=4$ & $n=3$ & $n=4$ & \\
\hline 0.2 & 1.118288834 & 1.118292667 & 1.118292550 & 1.118292655 & 1.118292654 \\
\hline 1.0 & 1.390507568 & 1.392394574 & 1.392344429 & 1.392351832 & 1.392351642 \\
\hline 4.0 & 1.831571465 & 1.907139788 & 1.903094833 & 1.903138679 & 1.903136945 \\
\hline 100.0 & $\begin{array}{lll}152.258 & 104 & 738\end{array}$ & $7.254843 \quad 292$ & $4.999 \quad 147 \quad 313$ & 4.999431869 & 4.999417545 \\
\hline 2000.0 & 3025.897452512 & $96.996 \quad 696 \quad 154$ & 13.387638106 & 13.388485529 & 13.388441701 \\
\hline
\end{tabular}
much more effectively than the perturbation series (2.1) unless $\beta$ is very small. For small or moderately large $\beta$, the Padé approximants $(1-\kappa)^{-1 / 2}(1+\kappa[6 / 6])$ and $(1-\kappa)^{-1 / 2}(1+\kappa[7 / 6])$ seem to be roughly equivalent to

TABLE II. Summation of the perturbation series (2.1) and (2.11) for the ground state energy $E^{(2)}(\beta)$ of the quartic anharmonic oscillator by effective characteristic polynomials (3.2) of degrees $n=3$ and $n=4$. 
TABLE III. Summation of the partial sums (3.8) and (3.9) of the perturbation series (2.1) and (2.11), respectively, for the ground state energy $E^{(2)}(\beta)$ of the quartic anharmonic oscillator by Padé approximants.

\begin{tabular}{|c|c|c|c|c|c|}
\hline \multirow[b]{2}{*}{$\beta$} & \multicolumn{2}{|c|}{ Perturbation series (2.1) } & \multicolumn{2}{|c|}{ Renormalized perturbation series ( 2.11 ) } & \multirow[b]{2}{*}{ Exact } \\
\hline & $1+\beta[6 / 6]$ & $1+\beta[7 / 6]$ & $\frac{1+\kappa[6 / 6]}{(1-\kappa)^{1 / 2}}$ & $\frac{1+\kappa[7 / 6]}{(1-\kappa)^{1 / 2}}$ & \\
\hline 0.2 & 1.118293197 & $1.118292 \quad 278$ & 1.118292655 & 1.118292655 & 1.118292654 \\
\hline 1.0 & 1.394169246 & 1.389827745 & 1.392351850 & 1.392352240 & 1.392351642 \\
\hline 4.0 & 1.995346220 & 1.635372857 & 1.903138615 & 1.903141052 & 1.903136945 \\
\hline 100.0 & 16.951874831 & -417.324934162 & $4.999 \quad 430 \quad 177$ & 4.999446387 & 4.999417545 \\
\hline 2000.0 & 311.178693279 & $-178 \quad 342.130076040$ & 13.388479935 & 13.388528318 & 13.388441701 \\
\hline
\end{tabular}

the effective characteristic polynomial of degree $n=4$. However, for $\beta=100$ or $\beta=2000$, the effective characteristic polynomial gives better results. Similarly, effective characteristic polynomials sum the perturbation series (2.1) more effectively than Padé approximants unless $\beta$ is very small.

The quality of our summation results can be improved considerably if some of the $n(n+3) / 2$ conditions needed for the construction of the unspecified coefficients $\mathcal{F}_{j}^{k}$ in Eq. (3.5) are provided by the renormalized strong coupling expansion (2.19). This is accomplished by substituting the renormalized strong coupling expansion (2.19) into the effective characteristic polynomial (3.5) according to

$$
\begin{aligned}
& \mathcal{P}_{n}\left(\sum_{\mu=0}^{\infty} \Gamma_{\mu}^{(m)}(1-\kappa)^{\mu}\right) \\
& =\sum_{\nu=0}^{n}\left(\sum_{\mu=0}^{\infty} \Gamma_{\mu}^{(m)}(1-\kappa)^{\mu}\right)^{\nu} \sum_{j=0}^{n-\nu} \mathcal{F}_{j}^{\nu} \kappa^{j}=0
\end{aligned}
$$

and by doing a Taylor series of the substituted polynomial up to powers of order $\ell-1$ in $1-\kappa$ :

$$
\begin{aligned}
\mathcal{P}_{n}\left(\sum_{\mu=0}^{\infty} \Gamma_{\mu}^{(m)}(1-\kappa)^{\mu}\right) \\
=\sum_{\lambda=0}^{\ell-1} \mathcal{B}_{\lambda}(1-\kappa)^{\lambda}+O\left((1-\kappa)^{\ell}\right) .
\end{aligned}
$$

We now determine the $n(n+3) / 2$ unspecified coefficients $\mathcal{F}_{j}^{\nu}$ of the effective characteristic polynomial $\mathcal{P}_{n}\left(E_{R}^{(m)}\right)$ by using $n(n+3) / 2-\ell$ conditions $\mathcal{A}_{t}=0$ with $0 \leq t \leq$ $[n(n+3) / 2]-\ell-1$ from the Taylor expansion (3.7) and $\ell$ conditions $\mathcal{B}_{\lambda}=0$ with $0 \leq \lambda \leq \ell-1$ from the Taylor expansion (3.11).
Hence, in Table IV we use for the construction of effective characteristic polynomials of degrees $n=3$ and $n=4$ not only the terms of the perturbation series $(2.11)$ but also also the leading terms (Table 10-19 of Ref. [35] or Table 2 of Ref. [37])

$$
\Gamma_{0}^{(2)}=0.73521401033121608077229144527689
$$

$$
\Gamma_{1}^{(2)}=0.27705567287994697140393753932961
$$

$$
\Gamma_{2}^{(2)}=-0.01117889720964502573425245405543
$$

of the renormalized strong coupling expansion (2.19) for the ground state energy $E^{(2)}(\beta)$ of the quartic anharmonic oscillator as input data. One, two, or three conditions, respectively, are supplied by the renormalized strong coupling expansion (2.19) at $\kappa=1$ according to Eqs. (3.12)-(3.14) and the remainder of the $n(n+3) / 2$ conditions are provided by the weak coupling expansion (2.11) at $\kappa=0$.

A comparison of the results in Tables II and IV shows that the inclusion of additional conditions at $\kappa=1$ greatly enhances the efficiency of effective characteristic polynomials, in particular in the strong coupling regime.

If we compare in Table IV the accuracy obtained by the different effective characteristic polynomials of degree $n=4$, we observe that the characteristic polynomial, which uses three conditions at $\kappa=1$, produces less accurate results than the polynomial, which uses two conditions at $\kappa=1$. Since this deterioration of accuracy is not

\begin{tabular}{|c|c|c|c|c|c|c|c|}
\hline$\beta$ & \multicolumn{2}{|c|}{ One condition at $\kappa=1$} & \multicolumn{2}{|c|}{ Two conditions at $\kappa=1$} & \multicolumn{2}{|c|}{ Three conditions at $\kappa=1$} & Exact \\
\hline 0.2 & 1.118292666 & 1.118292655 & $1.118 \quad 292701$ & $1.118 \quad 292654$ & $1.118 \quad 292567$ & $1.118 \quad 299592$ & 1.118292654 \\
\hline 1.0 & 1.392351487 & 1.392351664 & 1.392352104 & 1.392351628 & 1.392351432 & 1.392354530 & 1.392351642 \\
\hline 4.0 & $1.903 \quad 136 \quad 059$ & $1.903 \quad 137054$ & $1.903 \quad 137416$ & 1.903136925 & $\begin{array}{llll}1.903 & 136 & 871\end{array}$ & 1.903139480 & $1.903 \quad 136945$ \\
\hline 100.0 & $\begin{array}{llll}4.999 & 416 & 588\end{array}$ & $4.999 \quad 417669$ & $4.999 \quad 417589$ & $4.999417 \quad 543$ & $\begin{array}{llll}4.999 & 417 & 544\end{array}$ & $4.999422 \quad 556$ & 4.999417545 \\
\hline 2000.0 & 13.388441300 & 13.388441753 & 13.388441703 & 13.388441701 & 13.388441701 & 13.388454676 & 13.388441701 \\
\hline
\end{tabular}
observed in the case of the polynomials of degree $n=3$, we suspect that it is a consequence of rounding errors:

TABLE IV. Determination of the ground state energy $E^{(2)}(\beta)$ of the quartic anharmonic oscillator by effective characteristic polynomials of degrees $n=3$ and $n=4$. One, two, or three conditions, respectively, are supplied by the renormalized strong coupling expansion (2.18) according to Eqs. (3.12)-(3.14) and the remainder of the $n(n+3) / 2$ conditions are provided by the renormalized perturbation series $(2.10)$. 
The coefficients of the weak coupling expansion (2.11) are exact rational numbers, whereas the coefficients of the renormalized strong coupling expansion (2.19) are floating point numbers with a not completely known accuracy.

The sextic anharmonic oscillator is a much more demanding summation problem than the quartic anharmonic oscillator. Thus we also tried to determine the ground state energy $E^{(3)}(\beta)$ of the sextic anharmonic via effective characteristic polynomials, which were constructed from the terms of the perturbation series (2.11) and also from the leading terms (Table 10-22 of Ref. [35] or Table 3 of Ref. [37])

$$
\begin{aligned}
& \Gamma_{0}^{(3)}=0.625089812561 \\
& \Gamma_{1}^{(3)}=0.40765918068 \\
& \Gamma_{2}^{(3)}=-0.03151647679
\end{aligned}
$$

of the renormalized strong coupling expansion (2.19). However, these coefficients are at best accurate to nine places. Accordingly, we observed numerical instabilities when we tried to use up to three conditions at $\kappa=1$ for the construction of effective characteristic polynomials for $E^{(3)}(\beta)$. In order to avoid problems with numerical instabilities, we determined the leading coefficient $\Gamma_{0}^{(3)}$ of the renormalized strong coupling expansion (2.19) by a combined symbolic and numerical approach described in Ref. [78] with an accuracy of 35 places:

$$
\Gamma_{0}^{(3)}=0.62508981255837324773247118289895562 \text {. }
$$

In Table $\mathrm{V}$ effective characteristic polynomials for the ground state energy $E^{(3)}(\beta)$ were constructed using either all conditions at $\kappa=0$ from the renormalized perturbation series (2.10) or one condition from the renormalized strong coupling expansion (2.18) at $\kappa=1$ according to Eq. (3.18) and the remaining $n(n+3) / 2-1$ conditions from the renormalized perturbation series (2.10). The exact results in Table VII were obtained from Table II of Ref. [74].

The results in Table $\mathrm{V}$ show that effective characteristic polynomials apparently work even in the very demanding case of the ground state energy $E^{(3)}(\beta)$ of the sextic anharmonic oscillator. However, Table V also shows that the quality of the summation results is im- proved considerably -in particular for larger values of $\beta$-if in addition to the conditions at $\kappa=0$ a single condition at $\kappa=1$ is also included.

\section{TWO-POINT PADÉ APPROXIMANTS}

Let us assume that a function $f$ possess a formal power series

$$
f(z)=\sum_{\nu=0}^{\infty} a_{\nu} z^{\nu} .
$$

Its Padé approximant

$$
[\ell / m]_{f}(z)=P_{\ell}(z) / Q_{m}(z)
$$

is the ratio of two polynomials

$$
P_{\ell}(z)=p_{0}+p_{1} z+p_{2} z^{2}+\cdots+p_{l} z^{l}
$$

and

$$
Q_{m}(z)=1+q_{1} z+q_{2} z^{2}+\cdots+q_{l} z^{l}
$$

of degrees $\ell$ and $m$, respectively. The $\ell+m+1$ polynomial coefficients $p_{0}, p_{1}, \ldots, p_{\ell}$ and $q_{1}, q_{2}, \ldots, q_{m}$ are chosen in such a way that the Taylor series of the ratio $P_{\ell}(z) / Q_{m}(z)$ agrees as far as possible with the formal power series (4.1):

$$
f(z)-P_{\ell}(z) / Q_{m}(z)=O\left(z^{\ell+m+1}\right), \quad z \rightarrow 0 .
$$

If this relationship is rewritten as

$$
Q_{m}(z) f(z)-P_{\ell}(z)=O\left(z^{\ell+m+1}\right), \quad z \rightarrow 0,
$$

it leads to a system of $\ell+m+1$ linear equations for the coefficients of the polynomials $P_{\ell}(z)$ and $Q_{m}(z)$ [compare, for instance, Eq. (1.16) of Ref. [24]]. If this linear system has a solution, the Padé approximant can be represented as the ratio of two determinants that depend on the coefficients $a_{0}, a_{1}, \ldots, a_{\ell+m}$ of the formal power series (4.1) [compare, for instance, Eq. (1.27) of Ref. [24]].

Hence Padé approximants utilize only information from the expansion of $f$ around the origin up to a given

\begin{tabular}{|c|c|c|c|c|c|c|c|}
\hline \multirow[b]{2}{*}{$\beta$} & \multicolumn{3}{|c|}{ All conditions at $\kappa=0$} & \multicolumn{3}{|c|}{ One condition at $\kappa=1$} & \multirow[b]{2}{*}{ Exact } \\
\hline & $n=3$ & $n=4$ & $n=5$ & $n=3$ & $n=4$ & $n=5$ & \\
\hline 0.2 & 1.201633 & 1.173603 & 1.173904 & 1.174340 & 1.173849 & 1.173817 & 1.173889 \\
\hline 1.0 & 1.501199 & 1.433353 & 1.436143 & 1.437088 & 1.435419 & 1.435311 & 1.435625 \\
\hline 4.0 & 1.943220 & 1.823952 & 1.832385 & 1.832415 & 1.830118 & 1.829973 & 1.830437 \\
\hline 100.0 & 4.013010 & 3.691068 & 3.725831 & $3.718 \quad 544$ & 3.716713 & 3.716608 & 3.716974 \\
\hline 2000.0 & 8.347784 & 7.640275 & 7.723080 & 7.702576 & 7.701600 & 7.701545 & 7.701738 \\
\hline
\end{tabular}
order. This has far-reaching numerical consequences. As is well known, the numerical properties of power series of the type of Eq. (4.1) can frequently be improved consid-

TABLE V. Determination of the ground state energy $E^{(3)}(\beta)$ of the sextic anharmonic oscillator by effective characteristic polynomials of degrees $n=3, n=4$, and $n=5$. Either all $n(n+3) / 2$ conditions are provided by the renormalized perturbation series $(2.10)$ at $\kappa=0$ or one condition is supplied by the renormalized strong coupling expansion (2.18) at $\kappa=1$ according to Eq. (3.18) and the remaining $n(n+3) / 2-1$ conditions are provided by the renormalized perturbation series (2.10). 
erably by Padé approximants if $z$ is small or moderately large. However, Padé approximants usually do not accomplish much if $z$ is very large.

There are functions $f$ that also possesses an asymptotic series in powers of $1 / z$ :

$$
f(z) \sim \sum_{\nu=0}^{\infty} \frac{b_{\nu}}{z^{\nu}}, \quad z \rightarrow \infty .
$$

Asymptotic series of that kind are usually divergent for every $|z|<\infty$ and have to be summed. Also in this case Padé approximants to $f$ can be constructed by solving a system of linear equations. In this way, it is frequently possible to sum a divergent asymptotic series of the type of Eq. (4.7) efficiently if $z$ is not too small. However, Padé approximants, which only use information from the expansion (4.7) of $f$ around infinity, normally produce bad results if $z$ is very small.

The concept of Padé approximants can be generalized in such a way that information from both the origin as well as from infinity can be utilized simultaneously if a function $f$ possesses both a power series expansion (4.1) around the origin and an inverse power series expansion (4.7) around infinity. Such a two-point Padé approximant is again defined as the ratio of two polynomials $P_{\ell}(z)$ and $Q_{m}(z)$ of degrees $\ell$ and $m$, whose coefficients are defined by a system of $\ell+m+1$ linear equations. The only difference between a one-point and a two-point Padé approximant is that now some of the $\ell+m+1$ conditions are provided by the power series (4.1) and the remaining conditions are provided by the inverse power series (4.7).

A generalization to multipoint Padé approximants is also possible if the function $f$ possesses several power series expansions around the points $z_{0}, z_{1}, \ldots$,

$$
f(z)=\sum_{\nu=0}^{\infty} a_{\nu}^{(j)}\left(z-z_{j}\right)^{\nu}, \quad j=0,1, \ldots
$$

In this case, each expansion for $f$ contributes a certain number of conditions to the system of linear equations for the coefficients of the polynomials $P_{\ell}(z)$ and $Q_{m}(z)$.

Additional material on two-point and multipoint Padé approximants can for instance be found in Sec. 8 of [24], in Sec. 1.1 of Ref. [26], in Sec. 2.9 of Ref. [79], in Sec. VIII.2.1 of Ref. [80], and in Ref. [81] and [82].

The perturbation series $(2.1)$ for $E^{(m)}(\beta)$ is a weak coupling expansion around $\beta=0$ and the strong coupling expansion (2.2) is an expansion around infinity. Nevertheless, these two expansions cannot be used for the construction of two-point Padé approximants since the strong coupling expansion (2.2) is an expansion in $\beta^{-2 /(m+1)}$. Consequently, these two perturbation series have incompatible variables. In contrast, the two renormalized strong coupling expansions (2.11) and (2.19) for $E^{(m)}(\beta)$ are suited for the construction of two-point Padé approximants, since they are expansions in $\kappa$ and $1-\kappa$, respectively.

In all calculations involving two-point Padé approximants, we partition the ground state energy $E^{(m)}(\beta)$ into the prefactor $(1-\kappa)^{-1 / 2}$ and the renormalized ground state energy $E_{R}^{(m)}(\kappa)$ according to Eq. (2.8). We always retain the prefactor $(1-\kappa)^{-1 / 2}$ and only transform the perturbation expansions $(2.10)$ and $(2.18)$ for $E_{R}^{(m)}(\kappa)$ into two-point Padé approximants. This approach guarantees that the resulting two-point Padé approximants for $E^{(m)}(\beta)$ possess the correct asymptotic behavior as $\beta \rightarrow \infty$ according to Eq. (2.3).

Moreover, we only consider diagonal two-point Padé approximants with numerator and denominator polynomials of equal degrees. Hence we approximate $E_{R}^{(m)}(\kappa)$ by rational functions of the type

$$
\frac{P_{n}(\kappa)}{Q_{n}(\kappa)}=\frac{p_{0}+p_{1} \kappa+\cdots+p_{n} \kappa^{n}}{1+q_{1} \kappa+\cdots+q_{n} \kappa^{n}} .
$$

Such a two-point Padé approximant $P_{n}(\kappa) / Q_{n}(\kappa)$ contains $2 n+1$ unspecified coefficients $q_{0}, q_{1}, \ldots, q_{n}$ and $p_{1}$, $p_{2}, \ldots, p_{n}$ which have to be determined.

As in the case of the effective characteristic polynomials, we use MAPLE $[15,16]$ for both the construction and the solution of the system of $2 n+1$ linear equations. Let us assume that $2 n-\ell+1$ conditions are to be supplied by the weak coupling expansion (2.11) around $\kappa=0$ and $\ell$ conditions by the renormalized strong coupling expansion (2.19) around $\kappa=1$. Thus, for a fixed value of $n$ we compute symbolically the first $2 n-\ell+1$ terms of the Taylor expansion of $P_{n}(\kappa) / Q_{n}(\kappa)$ around $\kappa=0$,

$$
\frac{P_{n}(\kappa)}{Q_{n}(\kappa)}=\sum_{t=0}^{2 n-\ell} \mathcal{C}_{t} \kappa^{t}+O\left(\kappa^{2 n-\ell+1}\right),
$$

and the first $\ell$ terms of the Taylor expansion of $P_{n}(\kappa) / Q_{n}(\kappa)$ around $\kappa=1$,

$$
\frac{P_{n}(\kappa)}{Q_{n}(\kappa)}=\sum_{t=0}^{\ell-1} \mathcal{D}_{t}(1-\kappa)^{t}+O\left((1-\kappa)^{\ell}\right) .
$$

We obtain our system of $2 n+1$ linear equations by requiring that the first $2 n-\ell+1$ coefficients of the Taylor expansion (4.10) agree with the corresponding coefficients of the renormalized perturbation series (2.10),

$$
c_{t}^{(m)}=\mathcal{C}_{t}, \quad 0 \leq t \leq 2 n-\ell,
$$

and that the first $\ell$ coefficients of the Taylor expansion (4.11) agree with the corresponding coefficients of the renormalized strong coupling expansion (2.18)

$$
\Gamma_{\lambda}^{(m)}=\mathcal{D}_{\lambda}, \quad 0 \leq \lambda \leq \ell-1 .
$$

This system of $2 n+1$ linear equations for the coefficients $q_{0}, q_{1}, \ldots, q_{n}$ and $p_{1}, p_{2}, \ldots, p_{n}$ is solved symbolically.

Multiplication of the resulting two-point Padé approximant for $E_{R}^{(m)}(\kappa)$ by $(1-\kappa)^{-1 / 2}$ yields the corresponding two-point Padé approximant for $E^{(m)}(\beta)$. Finally, the numerical value of $\kappa$ is computed for a given value of $\beta$ by solving Eq. (2.5) numerically with the help of the MAPLE command fsolve and substituted in the twopoint Padé approximant, yielding an approximation to the numerical value of $E^{(m)}(\beta)$.

In Table VI the ground state energy $E^{(2)}(\beta)$ of the 
TABLE VI. Determination of the ground state energy $E^{(2)}(\beta)$ of the quartic anharmonic oscillator with the help of two-point Padé approximants $P_{n}(\kappa) / Q_{n}(\kappa)$ with $n=2$ and $n=3$. One, two, or three conditions, respectively, are supplied by the renormalized strong coupling expansion (2.18) at $\kappa=1$ according to Eqs. (3.12)-(3.14), and the remainder of the $2 n+1$ conditions are provided by the renormalized perturbation series (2.10).

\begin{tabular}{|c|c|c|c|c|c|c|c|}
\hline$\beta$ & \multicolumn{2}{|c|}{$\begin{array}{l}\text { One condition at } \kappa=1 \\
n=2 \quad n=3\end{array}$} & \multicolumn{2}{|c|}{$\begin{array}{ll}\text { Two conditions at } \kappa & =1 \\
n=2 & n=3\end{array}$} & \multicolumn{2}{|c|}{$\begin{array}{l}\text { Three conditions at } \kappa=1 \\
n=2 \quad n=3\end{array}$} & Exact \\
\hline 0.2 & $1.118 \quad 306 \quad 014$ & $1.118 \quad 293521$ & $1.118 \quad 281 \quad 115$ & $1.118 \quad 291928$ & 1.118303882 & $1.118 \quad 293 \quad 331$ & 1.118292654 \\
\hline 1.0 & $1.392 \quad 417302$ & 1.392360250 & 1.392334677 & 1.392349367 & 1.392356608 & 1.392352312 & 1.392351642 \\
\hline 4.0 & 1.903224982 & $1.903 \quad 151447$ & 1.903128332 & 1.903135463 & 1.903137904 & 1.903137115 & 1.903136945 \\
\hline 100.0 & $\begin{array}{llll}4.999 & 465 & 097\end{array}$ & $4.999 \quad 426 \quad 485$ & 4.999417016 & $4.999417 \quad 440$ & $4.999 \quad 417 \quad 552$ & $4.999 \quad 417 \quad 547$ & $4.999417 \quad 545$ \\
\hline 2000.0 & $13.388460 \quad 175$ & $13.388445 \quad 227$ & 13.38844167 & 13.388441695 & 13.388441701 & 13.388441701 & $13.388441 \quad 701$ \\
\hline
\end{tabular}

quartic anharmonic oscillator is approximated by twopoint Padé approximants $P_{n}(\kappa) / Q_{n}(\kappa)$ with $n=2$ and $n=3$. One, two or three conditions, respectively, are supplied by the renormalized strong coupling expansion (2.18) according to Eqs. (3.12)-(3.14), and the remaining of the $2 n+1$ conditions are provided by the renormalized perturbation series (2.10).

If we compare the results in Tables III and VI, we see that two-point Padé approximants give remarkably accurate results, which are clearly better than the results obtained by effective characteristic polynomials. The twopoint Padé approximants in Table VI are ratios of polynomials of degrees $n=2$ and $n=3$. Hence the perturbation expansions (2.10) and (2.18) have to provide only five and seven conditions, respectively. Ordinary Padé approximants are not nearly as effective. If we use the partial sums (3.9) with $m=2$ as input data for Wynn's $\epsilon$ algorithm [77], we find that the following Padé approximants to $E^{(2)}(\beta)$ have, for $\beta=2000$, approximately the same accuracy as the two-point Padé approximants in Table VI:

$$
\begin{aligned}
& \frac{1+\kappa[23 / 23]}{(1-\kappa)^{1 / 2}}=13.388441701389 \\
& \frac{1+\kappa[24 / 23]}{(1-\kappa)^{1 / 2}}=13.388441700575 .
\end{aligned}
$$

The second approximant containing the Padé approximant [24/23] needs all coefficients $c_{\nu}^{(2)}$ of the renormalized perturbation series (2.10) with $\nu \leq 48$.

The remarkable efficiency of two-point Padé approximants becomes also quite evident in the case of the ground state energy $E^{(3)}(\beta)$ of the sextic anharmonic oscillator. In Table VII we use for the construction of two-point Padé approximants $P_{n}(\kappa) / Q_{n}(\kappa)$ with $n=2$, $n=3$, and $n=4$ not only the terms of the perturbation series (2.11) but also also the leading terms of the renormalized strong coupling expansion (2.19) according to Eqs. (3.15)-(3.17). One, two, or three conditions, respectively, are supplied by the renormalized strong coupling expansion (2.19) at $\kappa=1$ and the remmainder of the $2 n+1$ conditions are provided by the weak coupling expansion (2.11) at $\kappa=0$. The exact results in Table VII were obtained from Table II of Ref. [74].

An effective characteristic polynomial of degree $n$ contains $n(n+3) / 2$ unspecified coefficients, whereas a twopoint Padé approximant $P_{n}(\kappa) / Q_{n}(\kappa)$ contains $2 n+1$ unspecified coefficients. Thus we can expect that twopoint Padé approximants should, for the same value of $n$, be less strongly affected by inaccuracies of the input data than effective characteristic polynomials. Moreover, the summation results obtained by effective characteristic polynomials are affected by inaccuracies of the input data not only via the solution of the system of linear equations but also via the numerical determination of the roots of the characteristic polynomials. Accordingly, we observed in Table VII at most some minor numerical instabilities in the case of the two-point Padé approximant $P_{n}(\kappa) / Q_{n}(\kappa)$ with $n=4$, which uses three conditions at $\kappa=1$.

It was mentioned before that the sextic anharmonic oscillator is a borderline case for Padé approximants: It can be shown rigorously that Padé approximants are able to sum the perturbation series (2.1). However, Padé approximants converge so slowly that they are computationally useless $[51,62,63]$. The renormalization scheme of Vinette and Cížek [74] does not change the situation substantially. If we use the partial sums (3.9) with $m=3$ as input data for Wynn's $\epsilon$ algorithm [77], we obtain, for $\beta=2 / 10$, the following approximants to $E^{(3)}(\beta)$ :

$$
\begin{aligned}
& \frac{1+\kappa[82 / 81]}{(1-\kappa)^{1 / 2}}=1.173988 \\
& \frac{1+\kappa[82 / 82]}{(1-\kappa)^{1 / 2}}=1.173890
\end{aligned}
$$

TABLE VII. Determination of the ground state energy $E^{(3)}(\beta)$ of the sextic anharmonic oscillator with the help of two-point

\begin{tabular}{|c|c|c|c|c|c|c|c|c|c|c|}
\hline \multirow[b]{2}{*}{$\beta$} & \multicolumn{3}{|c|}{ One condition at $\kappa=1$} & \multicolumn{3}{|c|}{ Two conditions at $\kappa=1$} & \multicolumn{3}{|c|}{ Three conditions at $\kappa=1$} & \multirow[b]{2}{*}{ Exact } \\
\hline & $n=2$ & $n=3$ & $n=4$ & $n=2$ & $n=3$ & $n=4$ & $n=2$ & $n=3$ & $n=4$ & \\
\hline 0.2 & 1.174710 & 1.174215 & 1.174057 & 1.173706 & 1.173818 & 1.173852 & 1.173949 & 1.173913 & 1.173901 & 1.173889 \\
\hline 1.0 & 1.436829 & 1.436162 & 1.435928 & 1.435513 & 1.435574 & 1.435594 & 1.435640 & 1.435632 & 1.435628 & 1.435625 \\
\hline 4.0 & 1.831603 & 1.830981 & 1.830755 & 1.830385 & 1.830412 & 1.830422 & 1.830441 & 1.830439 & 1.830437 & 1.830437 \\
\hline 100.0 & 3.717644 & 3.717297 & $3.717 \quad 168$ & 3.716969 & 3.716972 & 3.716973 & 3.716975 & 3.716975 & 3.716971 & 3.716974 \\
\hline 2000.0 & 7.702070 & 7.701899 & 7.701835 & 7.701738 & 7.701738 & 7.701738 & 7.701738 & 7.701738 & 7.701731 & 7.701738 \\
\hline
\end{tabular}
Padé approximants $P_{n}(\kappa) / Q_{n}(\kappa)$ with $n=2, n=3$, and $n=4$. One, two, or three conditions, respectively, are supplied by the renormalized strong coupling expansion (2.18) at $\kappa=1$ according to Eqs. (3.15)-(3.17), and the remainder of the $2 n+1$ conditions are provided by the renormalized perturbation series $(2.10)$. 
and for $\beta=2000$ we obtain the approximants

$$
\begin{aligned}
& \frac{1+\kappa[82 / 81]}{(1-\kappa)^{1 / 2}}=7.705569 \\
& \frac{1+\kappa[82 / 82]}{(1-\kappa)^{1 / 2}}=7.702155 .
\end{aligned}
$$

The approximants containing the Padé approximants $[82 / 82]$ need all coefficients $c_{\nu}^{(3)}$ of the renormalized perturbation series (2.10) with $\nu \leq 165$. Hence renormalization does not help much to speed up the prohibitively slow convergence of Padé approximants to the ground state energy $E^{(3)}(\beta)$ of the sextic anharmonic oscillator.

In contrast, the two-point Padé approximants in Table VII converge remarkably rapidly. For instance, the twopoint Padé approximant $P_{n}(\kappa) / Q_{n}(\kappa)$ with $n=2$, which uses only two conditions at $\kappa=0$ and three conditions at $\kappa=1$, reproduces $E^{(3)}(\beta)$ with an accuracy of at least four places in the interval $0.2 \leq \beta \leq 2000$.

The ground state energy $E^{(4)}(\beta)$ of the octic anharmonic oscillator is an extremely demanding summation problem. As mentioned before, it was proven rigorously by Graffi and Grecchi [64] that Padé approximants are not able to sum the perturbation series (2.1) in the octic case. In this context, Fig. 1-c of Ref. [74] is also quite instructive. The results published in Table VI of Ref. [34] indicate that the renormalization scheme of Vinette and Čížek [74] does not change the situation. Thus Padé approximants are not able to sum the renormalized perturbation series $(2.11)$ for the ground state energy $E^{(4)}(\beta)$ of the octic anharmonic oscillator.

If we want to apply two-point Padé approximants in the octic case, we are confronted with the problem that the coefficients $\Gamma_{n}^{(4)}$ of the renormalized strong coupling expansion (2.19) are at best accurate to seven places (compare Table 10-24 of Ref. [35] or Table 4 of Ref. [37]). In order to avoid problems with numerical instabilities, we determined the leading coefficient $\Gamma_{0}^{(4)}$ of the renormalized strong coupling expansion (2.19) by a combined symbolic and numerical approach described in Ref. [78] with an accuracy of 27 places:

$$
\Gamma_{0}^{(4)}=0.555130236018265911014365275 .
$$

In Table VIII we constructed two-point Padé approximants $P_{n}(\kappa) / Q_{n}(\kappa)$ with $2 \leq n \leq 6$. One condition was provided by the renormalized strong coupling expansion (2.18) at $\kappa=1$ according to Eq. (4.20) and the remaining $2 n$ conditions were provided by the renormalized perturbation series (2.10). The exact results in Table VIII were obtained from Table 5.10 of Ref. [75].

The results in Table VIII indicate that two-point Padé approximants using only a single additional condition at $\kappa=1$ are indeed able to produce convergent results for the ground state energy $E^{(4)}(\beta)$ of the octic anharmonic oscillator. More detailed investigations would certainly be highly desirable. So far, the bottleneck is the accurate computation of the coefficients $\Gamma_{n}^{(4)}$ of the renormalized strong coupling expansion (2.19) with $n \geq 1$.

\section{SUMMARY}

In this article we consider two unconventional summation methods for divergent perturbation expansions: effective characteristic polynomials, which are described in Sec. III, and two-point Padé approximants, which are described in Sec. IV. These two summation methods are designed for systems in which both a weak coupling expansion and a strong coupling expansion are available, since they can utilize simultaneously information from both expansions. Such a dual approach should at least in principle be capable of producing better results than a summation technique that uses only information from either the weak coupling or the strong coupling expansion.

In both cases, we accomplish a summation by constructing and solving systems of linear equations. We do this with the help of the symbolic manipulation language MAPLE $[15,16]$.

The quantum mechanical systems that we treat in this article are the anharmonic oscillators defined by the Hamiltonians (1.5). The perturbation series (2.1) for the ground state energy $E^{(m)}(\beta)$ of an anharmonic oscillator with anharmonicity $x^{2 m}$ is a so-called weak coupling expansion in $\beta$ that diverges quite strongly for every $\beta \neq 0$ and has to be summed. The ground state energy $E^{(m)}(\beta)$ possesses also the so-called strong coupling expansion (2.2), which is a power series in $\beta^{-2 /(m+1)}$ and converges if $\beta$ is sufficiently large.

The perturbation expansion (2.1) and the strong coupling expansion (2.2) have incompatible variables. Consequently, they cannot be used for the construction of effective characteristic polynomials or two-point Padé approximants, which use simultaneously information from the weak and the strong coupling expansion.

However, this aim can be accomplished with the help of alternative perturbation expansions for $E^{(m)}(\beta)$ that are based on a renormalization scheme worked out in full detail by Vinette and Č́žck [74]. This renormaliza-

TABLE VIII. Determination of the ground state energy $E^{(4)}(\beta)$ of the octic anharmonic oscillator with the help of two-point Padé approximants $P_{n}(\kappa) / Q_{n}(\kappa)$ with $2 \leq n \leq 6$. One condition is supplied by the renormalized strong coupling expansion (2.18) at $\kappa=1$ according to Eq. (4.20) and the remaining $2 n$ conditions are provided by the renormalized perturbation series (2.10).

\begin{tabular}{lcccccr}
\hline \hline$\beta$ & $n=2$ & $n=3$ & $n=4$ & $n=5$ & $n=6$ & Exact \\
\hline 0.2 & 1.245144 & 1.243919 & 1.243359 & 1.243039 & 1.242831 & 1.241028 \\
1.0 & 1.495243 & 1.494035 & 1.493478 & 1.493157 & 1.492947 & 1.491020 \\
4.0 & 1.825939 & 1.824882 & 1.824393 & 1.824110 & 1.823925 & 1.822180 \\
100.0 & 3.190961 & 3.190323 & 3.190027 & 3.189855 & 3.189743 & 3.188654 \\
2000.0 & 5.667524 & 5.667160 & 5.666991 & 5.666894 & 5.666829 & 5.666204 \\
\hline \hline
\end{tabular}


tion scheme is essentially the variable substitution (2.5), which maps the semi-infinite interval $[0, \infty)$ for $\beta$ onto the unit interval $[0,1)$ for the renormalized coupling constant $\kappa$. This renormalization scheme, which was originally designed for other purposes, also simplifies variational calculations in the strong coupling regime, as shown in Table I.

Our renormalization scheme partitions $E^{(m)}(\beta)$ according to Eq. $(2.8)$ into $(1-\kappa)^{-1 / 2}$, which behaves like $\beta^{1 /(m+1)}$ as $\beta \rightarrow \infty$ according to Eq. (2.9), multiplied by a renormalized ground state energy $E_{R}^{(m)}(\kappa)$. The renormalized energy $E_{R}^{(m)}(\kappa)$ can be expanded into a weak coupling expansion (2.10) or a strong coupling expansion (2.18).

Thus the ground state energy $E^{(m)}(\beta)$ possesses also the renormalized weak coupling expansion (2.11) [Eq. (5.3) of Ref. [34]], which is a strongly divergent power series in $\kappa$, and the renormalized strong coupling expansion (2.19) [Eq. (5.5) of Ref. [37]], which is a power series in $1-\kappa$ and apparently converges for all $\beta \in[0, \infty)$ (compare Secs. 6 and 7 of Ref. [37]).

The two renormalized perturbation series (2.11) and (2.19) are expansions in $\kappa$ and $1-\kappa$, respectively. Consequently, they have compatible variables and can be used for the construction of effective characteristic polynomials and and two-point Padé approximants.

Effective characteristic polynomials can also be used for the summation of the weak coupling expansions (2.1) and (2.11) for the ground state energy $E^{(2)}(\beta)$ of the quartic anharmonic oscillator. The results in Tables II and III indicate that they do this at least as effectively as Padé approximants. However, the results in Table IV show that the summation power of effective characteristic polynomials is greatly enhanced if also some conditions at $\kappa=1$ are included.

The principal advantages of simultaneously using conditions at $\kappa=0$ and at $\kappa=1$ are also demonstrated in Table V. Effective characteristic polynomials are apparently able to sum the renormalized weak coupling expansion (2.11) for the ground state energy $E^{(3)}(\beta)$ of the sextic anharmonic oscillator, albeit slowly. The inclusion of a single condition at $\kappa=1$ improves the summation results considerably, in particular in the strong coupling regime.

Even better summation results are obtained by twopoint Padé approximants. The results in Tables VI-VIII show that two-point Padé approximants, which simultaneously use information at $\kappa=0$ and at $\kappa=1$, clearly outperform ordinary Padé approximants that only use information at $\kappa=0$.

For example, ordinary Padé approximants are able to sum the renormalized strong coupling expansion (2.11) for the ground state energy $E^{(3)}(\beta)$ of the sextic anharmonic oscillator. However, Eqs. (4.16)-(4.19) show that Padé approximants to $E^{(3)}(\beta)$ converge too slowly to be practically useful. In contrast, the two-point Padé approximants $P_{n}(\kappa) / Q_{n}(\kappa)$ in Table VII produce already for $n=2$ remarkably accurate approximations to $E^{(3)}(\beta)$.

In the octic case, it was proven rigorously by Graffi and Grecchi [64] that Padé approximants do not converge. However, the results in Table VIII provide strong evidence that two-point Padé approximants, which only use a single condition at $\kappa=1$, converge to $E^{(4)}(\beta)$.

Our numerical results indicate that effective characteristic polynomials are less efficient than two-point Padé approximants. However, this does not imply that effective characteristic polynomials are necessarily less useful. It was already mentioned before that there are situations in which the symmetry of the exact Hamiltonian can be taken into account easily in the case of effective characteristic polynomials [43], but not in the case of two-point Padé approximants $[44,39]$.

\section{ACKNOWLEDGMENTS}

J.Č. would like to thank the Alexander von Humboldt Foundation for financial support, which made this cooperation possible. J.Č. would also like to thank Professor J. Ladik, Universität Erlangen-Nürnberg, for stimulating discussions and for his kind hospitality. The work, which led to this article, was supported in part by Natural Sciences and Engineering Research Council of Canada research grants, which made stays of E.J.W. and V.S. at the University of Waterloo possible. This support is gratefully acknowledged.
[1] Perturbation Theory and Its Application in Quantum Mechanics, edited by C. H. Wilcox (Wiley, New York, 1966).

[2] J. Killingbeck, Techniques of Applied Quantum Mechanics (Butterworths, London, 1975).

[3] C.M. Bender and S.A. Orszag, Advanced Mathematical Methods for Scientists and Engineers (McGraw Hill, New York, 1978).

[4] B. Simon, Bull. Am. Math. Soc. 24, 303 (1991).

[5] K.O. Friedrichs, Perturbation of Spectra in Hilbert Space (American Mathematical Society, Providence, 1965).

[6] T. Kato, Perturbation Theory for Linear Operators (Springer-Verlag, Berlin, 1976).

[7] V.P. Maslov, Théorie des Perturbations et Méthodes Asymptotiques (Dunod, Paris, 1972).
[8] F. Rellich, Perturbation Theory of Eigenvalue Problems (Gordon and Breach, New York, 1969).

[9] M. Reed and B. Simon, Methods of Modern Mathematical Physics IV: Analysis of Operators (Academic, New York, 1978).

[10] B.G. Adams, Algebraic Approach to Simple Quantum Systems (Springer-Verlag, Berlin, 1994).

[11] G.A. Arteca, F.M. Fernández, and E.A. Castro, Large Order Perturbation Theory and Summation Methods in Quantum Mechanics (Springer-Verlag, Berlin, 1990).

[12] Large-Order Behaviour of Perturbation Theory, edited by J.C. Le Guillou and J. Zinn-Justin (North-Holland, Amsterdam, 1990).

[13] K. Kumar, Perturbation Theory and the Nuclear Many Body Problem (North-Holland, Amsterdam, 1962). 
[14] Atoms in Strong Fields, edited by C.A. Nicolaides, C.W. Clark, and M.H. Nayfeh (Plenum, New York, 1990).

[15] B.W. Char, K.O. Geddes, G.H. Gonnet, B.L. Leong, M.B. Monagan, and S.M. Watt, Maple $V$ Language Reference Manual (Springer-Verlag, Berlin, 1991).

[16] B.W. Char, K.O. Geddes, G.H. Gonnet, B.L. Leong, M.B. Monagan, and S.M. Watt, Maple V Library Reference Manual (Springer-Verlag, Berlin, 1991).

[17] E. Borel, Leçons sur les Séries Divergentes (GautierVillars, Paris, 1928) [English translation by C.L. Critchfield and A. Vakar, Los Alamos Scientific Laboratory, Translation No. LA-6140-TR, 1975 (unpublished)].

[18] G.H. Hardy, Divergent Series (Clarendon, Oxford, 1949).

[19] B. Candelpergher, J.C. Nosmas, and F. Pham, Approche de la Résurgence (Hermann, Paris, 1993).

[20] J.-P. Ramis, Séries Divergentes et Théories Asymptotiques (Société Mathématique de France, Marseille, 1993).

[21] W. Balser, From Divergent Power Series to Analytic Functions (Springer-Verlag, Berlin, 1994).

[22] B. Shawyer and B. Watson, Borel's Method of Summability (Oxford University Press, Oxford, 1994).

[23] The Padé Approximant in Theoretical Physics, edited by G.A. Baker, Jr. and J.L. Gammel (Academic, New York, 1970).

[24] G.A. Baker, Jr., Essentials of Padé Approximants (Academic, New York, 1975).

[25] G.A. Baker, Jr. and P. Graves-Morris, Padé Approximants. Part I: Basic Theory (Addison-Wesley, Reading, MA, 1981).

[26] G.A. Baker, Jr. and P. Graves-Morris, Padé Approximants. Part II: Extensions and Applications (AddisonWesley, Reading, MA, 1981).

[27] C. Brezinski and J. van Iseghem, in Handbook of Numerical Analysis Vol. III, edited by P.G. Ciarlet and J.L. Lions (North-Holland, Amsterdam, 1994).

[28] E.J. Weniger, Comput. Phys. Rep. 10, 189 (1989).

[29] E.J. Weniger and J. Čížek, Comput. Phys. Commun. 59, 471 (1990).

[30] E.J. Weniger, J. Comput. Appl. Math. 32, 291 (1990).

[31] E.J. Weniger, J. Čížek, and F. Vinette, Phys. Lett. A 156, 169 (1991).

[32] C. Brezinski and M. Redivo Zaglia, Extrapolation Methods (North-Holland, Amsterdam, 1991).

[33] E.J. Weniger, Numer. Algorithms 3, 477 (1992).

[34] E.J. Weniger, J. Čížek, and F. Vinette, J. Math. Phys. 34, 571 (1993).

[35] E.J. Weniger, Habilitationsschriff, Universität Regensburg, 1994 (unpublished). Compressed .dvi and .ps files for this habilitation can be obtained via anonymous ftp from directory/pub/preprint/Weniger/habil of rchs 1. chemie .uni-regensburg.de

[36] E.J. Weniger, Int. J. Quantum Chem. 57, 256 (1996).

[37] E.J. Weniger, Ann. Phys. (N.Y.) (to be published).

[38] C.M. Bender and S. Boettcher, J. Math. Phys. 35, 1914 (1994).

[39] J. Čížek, A. Pellégatti, and J. Paldus, Int. J. Quantum Chem. 9, 987 (1975).

[40] B.G. Adams, Theor. Chim. Acta 73, 459 (1988).

[41] G.A. Baker, Jr. and J.D. Johnson, Phys. Rev. A 44, 2271 (1991).

[42] P. Bracken, Ph.D. thesis, University of Waterloo, 1994 (unpublished).

[43] P. Bracken and J. Čížek, Phys. Lett. A 194, 337 (1994).
[44] M. Takahashi, P. Bracken, J. Čížek, and J. Paldus, Int. J. Quantum Chem. 53, 457 (1995).

[45] P. Bracken and J. Čížek, Int. J. Quantum Chem. 53, 451 (1995).

[46] P. Bracken and J. Čížek, Int. J. Quantum Chem. (to be published).

[47] J. Čížek and P. Bracken, Int. J. Quantum Chem. (to be published).

[48] G.A. Baker, Jr., G.S. Rushbrooke, and H.E. Gilbert, Phys. Rev. 135, 1272 (1964).

[49] J. Čížek, J. Paldus, U.W. Ramgulam, and F. Vinette, Prog. Surf. Sci. 25, 17 (1987).

[50] J.W. Downing, J. Michl, J. Cížek, and J. Paldus, Chem. Phys. Lett. 67, 377 (1979).

[51] B. Simon, Ann. Phys. (N.Y.) 58, 76 (1970).

[52] C.M. Bender and T.T. Wu, Phys. Rev. 184, 1231 (1969).

[53] C.M. Bender and T.T. Wu, Phys. Rev. Lett. 27, 461 (1971).

[54] C.M. Bender and T.T. Wu, Phys. Rev. D 7, 1620 (1973).

[55] M. Dineykhan, G.V. Efimov, G. Ganbold, and S.N. Nedelko, Oscillator Representation in Quantum Physics (Springer-Verlag, Berlin, 1995).

[56] C. Itzykson and J.-B. Zuber, Quantum Field Theory (McGraw-Hill, New York, 1980).

[57] H. Kleinert, Path Integrals in Quantum Mechanics, Statistics, and Polymer Physics (World Scientific, Singapore, 1990).

[58] J.W. Negele and H. Orland, Quantum Many-Particle Systems (Addison-Wesley, Reading, MA, 1988).

[59] G. Parisi, Statistical Field Theory (Addison-Wesley, Reading, MA, 1988).

[60] L.S. Schulman, Techniques and Applications of Path Integration (Wiley, New York, 1981).

[61] J. Zinn-Justin, Quantum Field Theory and Critical Phenomena (Oxford University Press, Oxford, 1989).

[62] B. Simon, Int. J. Quantum Chem. 21, 3 (1982).

[63] J. Cížek and E. R. Vrscay, Int. J. Quantum Chem. 21, 27 (1982).

[64] S. Graffi and V. Grecchi, J. Math. Phys. 19, 1002 (1978).

[65] B. Simon, in Cargèse Lectures in Physics, edited by D. Bessis (Gordon and Breach, New York, 1972), Vol. 5.

[66] W. Janke and H. Kleinert, Phys. Rev. Lett. 75, 2787 (1995).

[67] F.T. Hioe and E.W. Montroll, J. Math. Phys. 16, 1945 (1975).

[68] F.T. Hioe, D. MacMillen, and E.W. Montroll, Phys. Rep. 43, 305 (1978).

[69] A.V. Turbiner and A.G. Ushveridze, J. Math. Phys. 29, 2053 (1988).

[70] F.M. Fernández, Phys. Lett. A 166, 173 (1992).

[71] R. Guardiola, M.A. Solís, and J. Ros, Nuovo Cimento B 107, 713 (1992).

[72] F.M. Fernández and R. Guardiola, J. Phys. A 26, 7169 (1993).

[73] J. Čížek and E.R. Vrscay, Int. J. Quantum Chem. Symp. 20, 65 (1986).

[74] F. Vinette and J. Čížek, J. Math. Phys. 32, 3392 (1991).

[75] H. Meißner, Ph.D. thesis, Universität Regensburg, 1995 (unpublished). Compressed .dvi and .ps files for this Ph.D. thesis can be obtained via anonymous ftp from directory/pub/preprint/Meissner.PhD of rchs1.chemie. uni-regensburg. de

[76] J. Stoer and R. Bulirsch, Introduction to Numerical Analysis (Springer-Verlag, New York, 1993). 
[77] P. Wynn, Math. Tables Aids Comput. 10, 91 (1956).

[78] O. Bludský, V. Špirko, and J. Čížek, J. Phys. Chem. 99, 15608 (1995).

[79] W.T. Thron and W.B. Jones, Appl. Numer. Anal. 4, 143 (1988).
[80] L. Lorentzen and H. Waadeland, Continued Fractions with Applications (North-Holland, Amsterdam, 1992).

[81] M.H. Gutknecht, Acta Appl. Math. 33, 165 (1993).

[82] S. Tokarzewski, J. Blawzdziewicz, and I. Andrianov, Numer. Algorithms 8, 313 (1994). 